\title{
A CONSTRUCTIVE PROOF OF THE POINCARÉ-BIRKHOFF THEOREM
}

\author{
LI YONG AND LIN ZHENGHUA
}

\begin{abstract}
In this paper, with the use of the homotopy method, a constructive proof of the Poincaré-Birkhoff theorem is given. This approach provides a global method for finding fixed points of area-preserving maps and periodic solutions of Duffing equations.
\end{abstract}

\section{INTRODUCTION}

As is well known, the Poincaré-Birkhoff theorem, proposed by Poincaré [38] in 1912 and proven by Birkhoff [7] in 1913, has been generalized by many authors, for example, Birkhoff [8-10], Jacobowitz [29], Ding [20], [21], Conley and Zehnder [12], [13] (about Arnold's conjecture [6]), Franks [24], [25] and Flucher [22]. Today, these notable results have become powerful tools in studying the dynamical systems and periodic solutions for some significant equations, for example Newton equations and Duffing equations. The related works can be found in [14, 16-20, 23, 25, 28, 29, 35] (and [15, 32, 34, 36] utilizing Moser's twist theorem [37]), and the references therein.

As an important aspect for applications, it is also significant to have a way of finding fixed points of area-preserving maps, such as Poincare maps of Newton equations and Duffing equations. Generally, such a map of an annulus which twists the boundary curves in opposite directions has at least two fixed points, and the sum of indices of fixed points on such an annulus equals zero (see [25]). These result in some difficulties in utilizing the classical numerical methods, for example, the Newton method and the continuation method. The main reasons are the local convergence of the former and the strong regularity of the latter, along the manifold of solutions.

The main aim of this paper is to present a global method of finding fixed points of area-preserving maps and periodic solutions of Duffing equations. More precisely, with use of the continuous homotopy method, we shall establish constructively the following Poincaré-Birkhoff theorem given by Jacobowitz [29] and Ding [21].

Let $A$ denote an annular region in $R^{2} \backslash\{0\}$, whose inner boundary $\Gamma_{1}$ and outer boundary $\Gamma_{2}$ are two disjoint closed simple curves. By $D_{i}$ we denote

Received by the editors February 9, 1994 and, in revised form, August 29, 1994.

1991 Mathematics Subject Classification. Primary 54H25, 58F05.

Key words and phrases. Constructive proof, the Poincaré-Birkhoff theorem, periodic solutions of Duffing equations. 
the bounded open region bounded by $\Gamma_{i}, i=1,2$. Hence, $A=\bar{D}_{2} \backslash D_{1}$, and $0 \in D_{1} \subset D_{2}$.

Theorem. Let $T: A \rightarrow T(A) \subset R^{2} \backslash\{0\}$ be an area-preserving homeomorphism. Suppose:

(i) $T$ has the polar coordinates

$$
r^{*}=f(r, \theta), \quad \theta^{*}=\theta+g(r, \theta)
$$

such that $g(r, \theta)>0$ on $\Gamma_{1}$ and $g(r, \theta)<0$ on $\Gamma_{2}$ where $f$ and $g$ are $C^{2}$ continuous and $2 \pi$-periodic in $\theta$.

(ii) There exists a continuous area-preserving map $T_{1}: \bar{D}_{2} \rightarrow R^{2}$ such that $\left.T_{1}\right|_{A}=T$, and $0 \in T_{1}\left(D_{1}\right)$.

Then $T$ has at least two fixed points in $A$.

Now let us make some comments.

(a) In our result, the inner boundary curve $\Gamma_{1}$ is not necessarily star shaped relative to the orgin.

(b) The continuous homotopy method which we use has been proposed originally by Keller [30], Kellogg, Li and Yorke [31] and Smale [39]. This method has played an important role in various problems of finding fixed points or zeros of maps; see, for example, $[1-3,11,26,27,33,40]$. There are three distinct, but interrelated, aspects of the homotopy method: (1) construction of the right homotopy map, (2) theoretical proof of global convergence for this homotopy map, and (3) tracking the zero curve of this homotopy map. The first aspect is to link the problem considered to a simpler one by considering a single parameter problem. The second aspect is a key to that method, because it guarantees the third aspect, that is, following paths to find the desired solutions. There have been some typical algorithms in the third aspect; see, for example, $[2,3$, 27].

Our constructive proof means for almost every point near the set $g(r, \theta)=$ 0 , there exists a $C^{1}$ path passing to that point such that two ends of this path are two distinct fixed points of the area-preserving map considered. Hence, by utilizing the usual path-following methods, one can find numerically two fixed points. In particular, when fixed points of the map are isolated, an end of that $C^{1}$ path leads to a fixed point with positive index and another end leads to one with negative index. Because the zeros of $g(r, \theta)=0$ are easier to determine than the fixed points of $T$ (for example, using the Newton method), we provide an effective global method of finding fixed points of area-preserving maps and periodic solutions of the Duffing equations. To our best knowledge, such global methods seem not to have been applied to this problem. Of course, the aspect of path-following can be carried out by utilizing the typical methods. This makes us focus our attention on the theoretical proof of the global convergence for the given homotopy (Newton-type), that is the constructive proof.

This paper is organized as follows. Section 2 is the main part, which exhibits such a "constructive proof" of the Poincaré-Birkhoff theorem. There it will be seen that for the singular case, $T$ has infinite fixed points in the set $g(r, \theta)=$ 0 , and finding fixed points is generally simpler; but for the nonsingular case, the problem becomes more complicated. In Section 3, we outline a general framework of applications to the Duffing equations. As stated above, we use the path-following algorithm given by Allgower and Georg [3]. Neverthless, we 
also provide an experimental example, which shows the efficiency of the reduced algorithm.

It should be pointed out that recently Alpern and Prasad [4, 5] have established a very interesting conbinational proof of the famous Conley-ZehnderFranks theorem. This approach is different from ours. In particular, our approach reduces finding fixed points of area-preserving maps into following solutions of suitable ordinary differential equations with initial values. Consequently, it is convenient to implement to applications.

\section{CONSTRUCTIVE PROOF}

In this section, we give a constructive proof of the Poincaré-Birkhoff theorem. From this proof we can obtain a global method of finding two fixed points for area-preserving maps. The following lemmas are vital to our discussion.

Lemma 1 (Sard's theorem). If $\phi: U \subset R^{m} \rightarrow R^{n}$ is a $C^{k}$ map on the open set $U$ with $k>\max \{0, m-n\}$, then the set of singular values of $\phi$ has $n$-dimensional Lebesgue measure zero. Consequently, the set of regular values of $\phi$ is dense in $R^{n}$.

Lemma 2 (the parametrized Sard theorem [11]). Let $V \subset R^{n}, U \subset R^{m}$ be open sets, and $\phi: V \times U \rightarrow R^{k}$ a $C^{r}$ map, where $r>\max \{0, m-k\}$. If $0 \in R^{k}$ is a regular value of $\phi$, then for almost all $a \in V, 0$ is a regular value of $\phi_{a}=\phi(a, \cdot)$.

Lemma 3 (Garcia and Zangwill [26]). Let $V \subset R^{n}$ be an open set and let $\phi: \bar{V} \times[0,1] \rightarrow R^{n}$ be a $C^{1}$ function and 0 a regular value on $V$. Then $H^{-1}=$ : $\{(x, t) \in V \times[0,1]: \phi(x, t)=0\}$ is a finite number of disjoint continuously differentiable paths. Any path is either a loop in $\bar{V} \times[0,1]$ or starts from $a$ boundary point of $\bar{V} \times[0,1]$ and ends at another boundary point of $\bar{V} \times[0,1]$.

Lemma 4 (Garcia and Zangwill [26]). Let $\phi: \bar{V} \times[0,1] \rightarrow R^{n}$ be a $C^{1}$ homotopy, and 0 a regular value on $V$, where $V \subset R^{n}$ is an open bounded set. Then each solution $x(s)$ of the initial value problem

$$
\frac{d x_{i}}{d s}=(-1)^{i+1} \operatorname{det} \phi_{i}^{\prime}, x_{i}(0)=x_{i 0} \quad(i=1, \ldots, n+1)
$$

determines a $C^{1}$ path in $\phi^{-1}(0)$, where $s$ is a parameter and

$$
\phi_{i}^{\prime}=\left(\phi_{x_{1}}, \ldots, \phi_{x_{i-1}}, \phi_{x_{i+1}}, \ldots, \phi_{x_{n+1}}\right) \text {. }
$$

Lemma 5. Under the assumptions of the Theorem, there exists an area-preserving map $T_{2}: \bar{D}_{2} \rightarrow R^{2}$ such that $\left.T_{2}\right|_{A}=T$ and $T_{2}(0)=0$.

Proof. By the assumption (ii), we have $P=T_{1}^{-1}(0) \in D_{1}$. Hence there exists a ball $B_{\delta}(P)$ with center $P$ and the radius $\delta$ such that $\bar{B}_{\delta}(P) \subset D_{1}$.

Let $P_{1} \in \partial B \cap \overline{O P}$, where $\overline{O P}$ denotes the line segment with end points 0 and $P$. Set $O_{1}=\frac{1}{2}\left[P+P_{1}\right]$. Choose a polar coordinate system $(\rho, \phi)$ in $R^{2}$ which has $O_{1}$ as its pole. With this coordinate system, define $S: R^{2} \rightarrow R^{2}$ by

$$
\rho^{*}=\rho, \quad \phi^{*}=\phi+h(\rho),
$$

where $h(\rho)$ is a $C^{\infty}$ function satisfying $h(\rho)=0$, for $\rho \geq \delta / 2 ; h(\rho)=\pi$, for $0<\rho \leq \delta / 4$. Obviously, $S$ is an area-preserving diffeomorphism, and

$$
S(0)=P,\left.\quad S\right|_{A}=\text { id (identity). }
$$


Define $T_{2}=T_{1} \circ S$. Then $T_{2}: \bar{D}_{2} \rightarrow R^{2}$ is an area-preserving homeomorphism, because it is a composition of such maps. Moreover,

$$
\left.T_{2}\right|_{A}=\left.T_{1} \circ S\right|_{A}=\left.T_{1}\right|_{A}=T, \quad T_{2}(0)=T_{1} \circ S(0)=T_{1}(P)=0,
$$

which completes the proof of the lemma.

For convenience, we also define a map $\Pi: R^{2} \backslash\{0\} \rightarrow\left(R_{+} \backslash\{0\}\right) \times[0,2 \pi]$ by

$$
\Pi(x, y)= \begin{cases}\left(\sqrt{x^{2}+y^{2}}, \arccos \frac{x}{\sqrt{x^{2}+y^{2}}}\right), & y \geq 0, \\ \left(\sqrt{x^{2}+y^{2}}, \pi+\arccos \frac{x}{\sqrt{x^{2}+y^{2}}}\right), & y<0 .\end{cases}
$$

Proof of the theorem. Let

$$
\begin{aligned}
& F(p)=T(p)-p=\Pi^{-1}(\bar{f}(\Pi(p)), g(\Pi(p))), \\
& J=\{p \in A: g(\Pi(p))=0\},
\end{aligned}
$$

where $\bar{f}=f(r, \theta)-r$. By Lemma 5 , without loss of generality, we may assume that the map $T_{1}$ satisfies $T_{1}(0)=0$. Hence, $T_{1}: \bar{D}_{2} \backslash\{0\} \rightarrow R^{2} \backslash\{0\}$. With the polar coordinate system, we can write $T_{1}$ in the following form:

$$
r^{*}=f_{1}(r, \theta), \quad \theta^{*}=\theta+g_{1}(r, \theta), \quad \text { in } \bar{D}_{2} \backslash\{0\},
$$

where $f_{1}$ and $g_{1}$ are continuous on $\bar{D}_{2} \backslash\{0\}$. Since $\left.T_{1}\right|_{A}=T$, we have

$$
f_{1}(r, \theta)=f(r, \theta), \quad g_{1}(r, \theta)=g(r, \theta), \quad \text { on } A .
$$

From the property of the polar coordinate system it follows that $f_{1}$ is $2 \pi$ periodic in $\theta$, and for some integer $k, g_{1}(r, \theta)=g(r, \theta)+2 k \pi$, on $\bar{D}_{2} \backslash\{0\}$. Since $g_{1}(r, \theta)$ is continuous and $\bar{D}_{2} \backslash\{0\}$ is connected, $k$ is independent of $(r, \theta)$. From (1) and the periodicity of $g(r, \theta)$, we obtain $k=0$, which implies that $g_{1}(r, \theta)$ is also $2 \pi$-periodic in $\theta$.

Define

$$
\tilde{T}(r, \theta)=\left(f_{1}(r, \theta), \theta+g_{1}(r, \theta)\right) \text { on }\left(R_{+} \backslash\{0\}\right) \times[0,2 \pi]
$$

and set

$$
\left.F(r, \theta)=\left(F_{1}(r, \theta), F_{2}(r, \theta)\right) \equiv T_{1}(r, \theta)-(r, \theta), \quad \text { on }\left(R_{+}\right\rfloor\{0\}\right) \times[0,2 \pi] \text {. }
$$

We shall complete the proof by the following two steps.

$1^{o}$. Singular case. For all $(r, \theta) \in \Pi A$, the Jacobian $F^{\prime}(r, \theta)$ is singular. Take a simply connected region $\Omega \subset D_{2}$ such that $\bar{D}_{1} \subset \Omega$ and $\partial \Omega \subset J$. Indeed, by (i) there exists a bounded and connected branch $\Omega_{1} \subset R^{2} \backslash J$ such that

$$
0 \in \Omega_{1} \text { and } \partial \Omega_{1} \subset J .
$$

Thus $D_{1}$ is a region. Let $K$ denote the set of all closed simple curves $\nu$ lying in $\Omega_{1}$. By $D(\nu)$ we denote the bounded region bounded by $\nu$. Set

$$
\Omega=\bigcup\left\{D(\nu): \nu \text { lies in } \Omega_{1}\right\} .
$$

Then $\Omega$ is the desired simply connected region.

We claim:

$$
F_{1}(r, \theta) \equiv 0, \text { on } \partial \Omega \text {. }
$$


By (i), there exists $\left(r_{0}, \theta_{0}\right) \in \Pi A$ such that rank $F^{\prime}\left(r_{0}, \theta_{0}\right)=1$. Hence by Lemma 1 , almost every $r \in \operatorname{range}\left(F_{1}\right)$ is a regular value of $F_{1}$. First, we prove

$$
F_{1}(r, \theta) \equiv c \text { (a constant), on } \partial \Omega \text {. }
$$

If not, then by the continuity of $F_{1}, F_{1}(\Pi \partial \Omega)$ is an interval in $R$. Let $S_{1}$ denote the set of all regular values in $F_{1}(\Pi \partial \Omega)$. Since $\partial \Omega$ is bounded and closed, and $F_{1}$ is $C^{1}, S_{1}$ is open in $R$, and consequently, $S_{2}=F_{1}^{-1}\left(\Pi S_{1}\right)$ is open in the relative topology of $\Pi \partial \Omega$. By the inverse function theorem, for each $\left(r_{0}, \theta_{0}\right) \in S_{2}$, there exists a positive number $\eta=\eta\left(r_{0}, \theta_{0}\right)$ such that on $\left[\theta_{0}-\eta, \theta_{0}+\eta\right]$, the equation

$$
F_{1}(r, \theta)=F_{1}\left(r_{0}, \theta_{0}\right)
$$

has a unique solution $(r(\theta), \theta)$ satisfying

$$
r\left(\theta_{0}\right)=r_{0}, \quad\left|r(\theta)-r_{0}\right| \leq \eta,
$$

if $F_{1 r}\left(r_{0}, \theta_{0}\right) \neq 0$; or on $\left[r_{0}-\eta, r_{0}+\eta\right]$, equation (3) has a unique solution $(r, \theta(r))$ satisfying

$$
\theta\left(r_{0}\right)=\theta_{0}, \quad\left|\theta(r)-\theta_{0}\right| \leq \eta,
$$

if $F_{1 \theta}\left(r_{0}, \theta_{0}\right) \neq 0$. We can choose a small $\eta$ such that $F_{1 r} \neq 0$, on $I_{\eta}=$ $\left[r_{0}-\eta, r_{0}+\eta\right] \times\left[\theta_{0}-\eta, \theta_{0}+\eta\right]$; or $F_{1 \theta} \neq 0$ on $I_{\eta}$. For definiteness, let us assume it is the former case. Since $F^{\prime}$ is singular, there exists a continuous function $\lambda(r, \theta)$ defined on $I_{\eta}$ such that

$$
\left(F_{2 r}, F_{2 \theta}\right)=\lambda(r, \theta)\left(F_{1 r}, F_{1 \theta}\right) \text { on } I_{\eta} \text {. }
$$

From (4) and

$$
0 \equiv \frac{d F_{1}(r(\theta), \theta)}{d \theta}=F_{1 r} \frac{d r}{d \theta}+F_{1 \theta}
$$

it follows that

$$
\frac{d F_{2}(r(\theta), \theta)}{d \theta}=F_{2 r} \frac{d r}{d \theta}+F_{2 \theta}=\lambda(r(\theta), \theta) \frac{d F_{1}(r(\theta), \theta)}{d \theta}=0, \text { on } I_{\eta} .
$$

Hence on $I_{\eta} \cap \partial \Omega$,

$$
\left(F_{1}(r, \theta), F_{2}(r, \theta)\right) \equiv\left(F_{1}\left(r_{0}, \theta_{0}\right), F_{2}\left(r_{0}, \theta_{0}\right)\right)=\left(F_{1}\left(r_{0}, \theta_{0}\right), 0\right),
$$

which implies

$$
(r(\theta), \theta) \in \partial \Omega \text {. }
$$

Set $I(\eta(r, \theta))=(r-\eta(r, \theta), r+\eta(r, \theta)) \times(\theta-\eta(r, \theta), \theta+\eta(r, \theta))$. Since the set of all members in $\left\{I(\eta(r, \theta)):(r, \theta) \in S_{2}\right\}$ disjoint from each other is countable, by (5) $S_{1}$ is also countable, which contradicts the openess of $S_{1}$. Therefore (2) holds.

Now we prove that $c=0$. If $c \neq 0$, then from $F_{1}(r, \theta) \equiv c$ on $\partial \Omega$, we have $f(r, \theta)=r+c$, on $\Pi \partial \Omega$. Note

$$
g(r, \theta) \equiv 0, \text { on } \Pi \partial \Omega .
$$

Therefore,

$$
\Pi \tilde{T}(\Omega)=\left\{\left(r^{*}, \theta^{*}\right): r^{*}=r+c, \theta^{*}=\theta^{*},\left(r, \theta^{*}\right) \in \Pi \Omega\right\}=G(\Pi \Omega),
$$


where $G(r, \theta)=(r+c, \theta)$. Hence

$$
\Pi \tilde{T}(\Omega)=G(\Pi \Omega) .
$$

Given any $\Delta S=\left\{(r, \theta): 0<r_{1} \leq r \leq r_{2}, \theta_{1} \leq \theta \leq \theta_{2}\right\} \subset \Pi \Omega$, we have

$$
G(\Delta S) \subset \Pi T_{1}(\Omega),
$$

$$
\begin{aligned}
\operatorname{meas}[G(\Delta S)] & =\frac{\pi}{360}\left(\theta_{2}-\theta_{1}\right)\left[\left(r_{2}+c\right)^{2}-\left(r_{1}+c\right)^{2}\right] \\
& =\frac{\pi}{360}\left(\theta_{2}-\theta_{1}\right)\left(r_{2}-r_{1}\right)\left(r_{2}+r_{1}+2 c\right) \\
& =\frac{2 \pi c}{360}\left(\theta_{2}-\theta_{1}\right)\left(r_{2}-r_{1}\right)+\operatorname{meas}(\Delta S) .
\end{aligned}
$$

Thus, when $c \neq 0$, we obtain

$$
\operatorname{meas}\left[T_{1}(\Omega)\right]=\operatorname{meas}\left[\Pi T_{1}(\Omega)\right]=\operatorname{meas}[G(\Pi \Omega)] \neq \operatorname{meas}(\Pi \Omega)=\operatorname{meas}(\Omega),
$$

a contradiction. This proves that $c=0$, and consequently, every point in $\partial \Omega$ is a fixed point of $T$.

$2^{\circ}$ Regular case. For some $a_{0} \in \Pi A, F^{\prime}\left(a_{0}\right)$ is nonsingular. We claim that for some $\left(r_{0}, \theta_{0}\right) \in \Pi A$,

$$
f\left(r_{0}, \theta_{0}\right)-r_{0} \neq 0 \text {. }
$$

If not, then

$$
f(r, \theta)-r \equiv 0, \text { on } \Pi A \text {. }
$$

Choose any $\bar{\theta} \in g(\Pi A)$ and consider the equation

$$
f(r, \theta)-r \equiv 0, g(r, \theta)-\bar{\theta} \equiv 0 .
$$

Denote $J(\bar{\theta})=\left\{\Pi^{-1}(r, \theta) \in A: g(r, \theta)-\bar{\theta}=0\right\}$. Hence

$$
\begin{aligned}
& \left(f_{r}-1\right) d r+f_{\theta} d \theta \equiv 0, \text { on } \Pi A, \\
& g_{r} d r+g_{\theta} d \theta \equiv 0, \text { on } \Pi J(\bar{\theta}) .
\end{aligned}
$$

Therefore,

$$
\operatorname{det}\left(\begin{array}{cc}
f_{r}-1 & f_{\theta} \\
g_{r} & g_{\theta}
\end{array}\right)=0 \text {, on } \Pi J(\bar{\theta}),
$$

which leads to a contradiction, because $\bar{\theta}$ is arbitrary.

By Lemma 1 and the above claim, almost every point $(\bar{r}, \bar{\theta}) \in\left(F_{1}(\Pi A)\right.$, $\left.\left(\sigma_{1}, \sigma_{2}\right)\right)$ satisfying $\bar{r} \neq 0$ is a regular value of $\left(F_{1}, F_{2}\right)$, where $\sigma_{1}=$ $\max _{p \in \Gamma_{2}} g(r(p), \theta(p))<\min _{p \in \Gamma_{1}} g(r(p), \theta(p))=\sigma_{2}$. For such a regular value $(\bar{r}, \bar{\theta})$, set

$$
T_{\bar{\theta}}: r^{*}=f_{1}(r, \theta), \quad \theta^{*}=\theta+g_{1}(r, \theta)-\bar{\theta}, \quad \bar{\theta} \in\left(\sigma_{1}, \sigma_{2}\right) .
$$

Obviously, $T_{\bar{\theta}}$ is an area-preserving homeomorphism defined on $\Pi\left(D_{2} \backslash\{0\}\right)$. Set

$$
F_{\bar{\theta}}(r, \theta)=\left(f_{1}(r, \theta)-r, g_{1}(r, \theta)-\bar{\theta}\right) ;
$$

and define a homotopy map $H: \Pi \bar{A} \times \Pi \bar{A} \times[-\lambda(\bar{r}), 1] \rightarrow R^{2}$ by

$$
H_{\bar{\theta}}\left(p_{0}, p, \lambda\right)=F_{\bar{\theta}}(p)-(1-\lambda) F_{\bar{\theta}}\left(p_{0}\right),
$$


where $p=(r, \theta), F_{\bar{\theta}}\left(p_{0}\right)=(\bar{r}, 0), p_{0} \in F_{\bar{\theta}}^{-1}(\bar{r}, 0)$, and $\lambda(\bar{r})$ is a positive constant such that

$$
\frac{1}{2}|\bar{r}| \lambda(\bar{r}) \geq \max \left\{r: r=\sqrt{x^{2}+y^{2}},(x, y) \in A \cup T(A)\right\} .
$$

Notice that if for some $p_{0}=(r, \theta) \in \Pi A, F^{\prime}(r, \theta)$ is nonsingular, then

$$
\frac{\partial H}{\partial(r, \theta)}=-(1-\lambda) F^{\prime}(r, \theta)
$$

is also nonsingular for $\lambda<1$. Set $V=\left\{P=(r, \theta) \in F^{-1}(F(\Pi A)):(\bar{r}, \bar{\theta})\right.$ is a regular value of $F$ on $\Pi A$ and $\left.\bar{r} \neq 0, \bar{\theta} \in\left(\sigma_{1}, \sigma_{2}\right)\right\}$. By Lemma 2 , for almost all $p_{0} \in V, 0$ is a regular value of $H_{p_{0}} \equiv H\left(p_{0}, \cdot\right)$, for $\lambda \in[-\lambda(\bar{r}), 1)$. Denote by $V_{1}$ all such regular values.

Choose any $(\bar{r}, \bar{\theta})$ such that $F^{-1}(\bar{r}, \bar{\theta}) \subset V_{1}$. Let $x_{1}=r, x_{2}=\theta, x_{3}=\lambda$ and $P=\left(x_{1}, x_{2}\right)$. Then by Lemmas 3, 4, for each $p_{0}=\left(r_{0}, \theta_{0}\right) \in F^{-1}(\bar{r}, \bar{\theta})$, the solution $\left(P\left(s, p_{0}\right), \lambda\left(s, p_{0}\right)\right)$ of the initial value problem

$$
\begin{aligned}
& \frac{d x_{i}}{d s}=(-1)^{i+1} \operatorname{det} H_{i}^{\prime}, \quad i=1,2,3, \\
& (P(0), \lambda(0))=\left(p_{0}, 0\right)
\end{aligned}
$$

determines a $C^{1}$ path $\left(P\left(s, p_{0}\right), \lambda\left(s, p_{0}\right)\right)$ in $H_{p_{0}}^{-1}(0)$, where

$$
\begin{aligned}
& H_{1}^{\prime}=\left(H_{x_{2}}^{\prime}, H_{x_{3}}^{\prime}\right)=\operatorname{det}\left(\begin{array}{cc}
f_{\theta} & \bar{r} \\
g_{\theta} & 0
\end{array}\right)=-\bar{r} g_{\theta}, \\
& H_{2}^{\prime}=\left(H_{x_{1}}^{\prime}, H_{x_{3}}^{\prime}\right)=\operatorname{det}\left(\begin{array}{cc}
f_{r}-1 & \bar{r} \\
g_{r} & 0
\end{array}\right)=-\bar{r} g_{r}, \\
& H_{3}^{\prime}=\left(H_{x_{1}}^{\prime}, H_{x_{2}}^{\prime}\right)=\operatorname{det}\left(\begin{array}{cc}
f_{r}-1 & f_{\theta} \\
g_{r} & g_{\theta}
\end{array}\right)\left(=F^{\prime}(p)\right) ;
\end{aligned}
$$

and

$$
\begin{aligned}
H^{-1}(0)= & \left\{p=(r, \theta) \in \Pi A: H\left(p_{0}, p, \lambda\right)=0,\right. \\
& \left.\lambda \in[-\lambda(\bar{r}), 1), p_{0} \in F^{-1}(\bar{r}, \bar{\theta})\right\} \\
= & \left\{P\left(s, p_{0}\right) \in \Pi A: \lambda\left(s, p_{0}\right) \in[-\lambda(\bar{r}), 1), p_{0} \in F^{-1}(\bar{r}, \bar{\theta})\right\} .
\end{aligned}
$$

Since $\bar{\theta} \in\left(\sigma_{1}, \sigma_{2}\right)$ and

$$
H\left(p_{0}, P\left(s, p_{0}\right), \lambda\left(s, p_{0}\right)\right)=0,
$$

we have

$$
\begin{aligned}
\tilde{g}_{1}\left(r\left(s, p_{0}\right), \theta\left(s, p_{0}\right)\right) & \equiv g_{1}\left(r\left(s, p_{0}\right), \theta\left(s, p_{0}\right)\right)-\bar{\theta} \\
& =\left(1-\lambda\left(s, p_{0}\right)\right) \cdot 0=0, \text { for }-\lambda(\bar{r}) \leq \lambda\left(s, p_{0}\right) \leq 1 .
\end{aligned}
$$

Hence,

$$
J[\bar{\theta}] \equiv\left\{(r, \theta):(r, \theta)=\left(r\left(s, p_{0}\right), \theta\left(s, p_{0}\right)\right)\right\} \subset \Pi A .
$$

By Lemma 3, $\left(P\left(s, p_{0}\right), \lambda\left(s, p_{0}\right)\right)$ is either a loop in $\Pi \bar{A} \times[-\lambda(\bar{r}), 1]$ or starts from boundary points of $\Pi \bar{A} \times[-\lambda(\bar{r}), 1]$ and ends at boundary points of $\Pi \bar{A} \times$ $[-\lambda(\bar{r}), 1]$. We shall prove that the former is impossible. Let $\left(P\left(s, p_{0}\right), \lambda\left(s, p_{0}\right)\right)$ be a loop. By $\Omega\left(p_{0}\right)$ we denote the bounded region bounded by $P\left(s, p_{0}\right)$. We claim

$0 \in \Omega\left(p_{0}\right)$ and the orbit $\left\{P\left(s, p_{0}\right)\right\}$ is star shaped about the origin 0. 
First, $\left\{P\left(s, p_{0}\right)\right\}$ is star shaped about the origin 0 . Set $P(s)=P\left(s, p_{0}\right)=$ $(r(s), \theta(s)), \lambda(s)=\lambda\left(s, p_{0}\right)$. If not, then there would exist $s_{0} \in R$ such that $\theta\left(s_{0}\right)$ is minimal and $d \theta /\left.d s\right|_{s=s_{0}}=0$. Since $d \theta / d s=g_{r} \bar{r}$, we see $g_{r}\left(r\left(s_{0}\right), \theta\left(s_{0}\right)\right)=0$. Notice that the line $\theta=\theta\left(s_{0}\right)$ is a tangent of $(r(s), \theta(s))$ at $\left(r\left(s_{0}\right), \theta\left(s_{0}\right)\right)$. By the area-preserving property of $T_{\bar{\theta}}$, we have

$$
\operatorname{det} T_{\bar{\theta}}^{\prime}(r, \theta)=\operatorname{det}\left(\begin{array}{cc}
f_{r} & f_{\theta} \\
g_{r} & 1+g_{\theta}
\end{array}\right)=r .
$$

Hence for any $(r, \theta) \in \Pi A$,

$$
\Delta r^{*} \Delta \theta^{*}=\left.\operatorname{det}\left(\begin{array}{cc}
f_{r} & f_{\theta} \\
g_{r} & 1+g_{\theta}
\end{array}\right)\right|_{(r+\xi \Delta r, \theta+\xi \Delta \theta)} \Delta r \Delta \theta=(r+\xi \Delta r) \Delta r \Delta \theta,
$$

whenever $|\Delta r|,|\Delta \theta|$ are sufficiently small. Since for $s_{1}, s_{2} \in R$, along the curve $\{P(s)\}$, we get

$$
\Delta r^{*} \Delta \theta^{*}=\Delta r^{*} \Delta \theta=f_{r}(r+\xi \Delta r, \theta+\xi \Delta \theta) \Delta r \Delta \theta
$$

From

$$
\begin{aligned}
& r^{*}(s)=f(r(s), \theta(s))=(1-\lambda(s)) \bar{r}+r(s), \\
& \theta^{*}(s)=g(r(s), \theta(s))-\bar{\theta}=0, \\
& \left.\operatorname{rank}\left(H_{r}, H_{\theta}, H_{\lambda}\right)\right|_{(r(s), \theta(s), \lambda(s)), \lambda(s)<1}=2,
\end{aligned}
$$

it follows that

$$
\begin{aligned}
& g_{\theta}\left(r\left(s_{0}\right), \theta\left(s_{0}\right)\right) \neq 0,\left.\frac{d \theta^{*}(s)}{d s}\right|_{s=s_{0}}=\left.\frac{d \theta(s)}{d s}\right|_{s=s_{0}}=0, \\
& \left.\frac{d r^{*}(s)}{d s}\right|_{s=s_{0}}=\left.f_{r} \frac{d r(s)}{d s}\right|_{s=s_{0}} \cdot
\end{aligned}
$$

Because $P(s)$ is a loop, we can choose such an $s_{0}$ to possess the following property:

(P) For every $\epsilon>0$, meas $\left\{\theta(s): s \in\left[s_{0}-\epsilon, s_{0}+\epsilon\right]\right\}>0$.

Then using (10),(11), and (13) yields

$$
0 \neq r\left(s_{0}\right)=f_{r}\left(r\left(s_{0}\right), \theta\left(s_{0}\right)\right)\left[1+g_{\theta}\left(r\left(s_{0}\right), \theta\left(s_{0}\right)\right)\right]=f_{r}\left(r\left(s_{0}\right), \theta\left(s_{0}\right)\right) .
$$

Then

$$
g_{\theta}\left(r\left(s_{0}\right), \theta\left(s_{0}\right)\right)=0
$$

Conseqently,

a contradiction.

$$
\left.\operatorname{rank}\left(H_{r}, H_{\theta}, H_{\lambda}\right)\right|_{\left(r\left(s_{0}\right), \theta\left(s_{0}\right), \lambda\left(s_{0}\right)\right)}=1,
$$

It is clear that $0 \in \Omega\left(p_{0}\right)$. By the claim and (12), we have

$$
T_{\bar{\theta}}\left[\Omega\left(p_{0}\right)\right] \subset \Omega\left(p_{0}\right), \text { if } \bar{r}<0 ;
$$

or

$$
T_{\bar{\theta}}\left[\Omega\left(p_{0}\right)\right] \supset \Omega\left(p_{0}\right), \text { if } \bar{r}>0 .
$$

Notice that $\lambda(s) \not \equiv 1$. Therefore from (14) and (15) it follows that

$$
\operatorname{meas}\left[T_{\bar{\theta}}\left(\Omega\left(p_{0}\right)\right)\right] \neq \operatorname{meas}\left(\Omega\left(p_{0}\right)\right) \text {, }
$$

a contradiction. This shows that $\{(P(s), \lambda(s))\}$ is not a loop. 
By (6),(9) and (12), we get

$$
\lambda(s) \geq-\frac{1}{2} \lambda(\bar{r}), \text { for } s \in R
$$

Hence $\Gamma\left(p_{0}\right)=\{(P(s), \lambda(s)): \lambda(s) \leq 1\}$ starts from the points in $\Pi A \times\{1\}$ and ends at the points in $\Pi A \times\{1\}$. Set

$$
S_{ \pm}=\left\{q \in \Pi A: P\left(s, p_{0}\right) \text { leads to } q \text {, as } \lambda(s) \rightarrow 1, s \in R_{ \pm}\right\} .
$$

We claim

$$
S_{+} \cap S_{-}=\varnothing \text {. }
$$

If not, then $S_{+} \cap S_{-} \neq \varnothing$. By the above arguments, for each $s \in R$ with $\lambda(s)<1, \theta(s)$ is not an extreme value. Otherwise, for some $s_{0} \in R, \theta\left(s_{0}\right)$ is an extreme value. Then for any interval $I$ containing $s_{0}$, the property $(\mathbf{P})$ does not hold. Therefore

$$
\theta(s) \equiv \text { a constant, for } \lambda(s)<1 .
$$

Since $\left.\operatorname{rank}\left(H_{r}, H_{\theta}, H_{\lambda}\right)\right|_{(p(s), \lambda(s)), \lambda(s)<1}=2$, we have

$$
\frac{d r(s)}{d s} \neq 0, \text { for } \lambda(s)<1
$$

This shows that $S_{+} \cap S_{-}=\varnothing$, a contradiction. Hence when $\lambda(s)<1$,

$$
\frac{d \theta(s)}{d s} \geq 0 \quad\left(\text { or } \frac{d \theta(s)}{d s} \leq 0\right) \text {. }
$$

From (17) and (18) it follows that there exist $\theta_{1}, \theta_{2} \in R$ with $\left|\theta_{1}-\theta_{2}\right|=2 \pi$ such that

$$
\theta(s) \rightarrow \begin{cases}\theta_{1}, & \text { as } \lambda(s) \rightarrow 1, s \in R_{+}, \\ \theta_{2}, & \text { as } \lambda(s) \rightarrow 1, s \in R_{-} .\end{cases}
$$

Then $\Gamma\left(p_{0}\right)$ bounds a bounded region $\Omega\left(p_{0}\right)$. Notice that for each $\theta \in$ $\left(\min \left\{\theta_{1}, \theta_{2}\right\}, \max \left\{\theta_{1}, \theta_{2}\right\}\right),\{(r, \theta): r \geq 0\} \cap \Gamma\left(p_{0}\right)$ contains only one point. Thus by (12), meas $\left(T_{\bar{\theta}}\left(\Omega\left(p_{0}\right)\right)\right) \neq \operatorname{meas}\left(\Omega\left(p_{0}\right)\right)$. This contradiction shows that (17) is true.

Using (12), we know that every point $p \in S_{+} \cup S_{-}$is a fixed point of $T_{\bar{\theta}}$. By (17), $T_{\bar{\theta}}$ has at least two fixed points in $A$. In particular, when $S_{+}$and $S_{-}$are isolated sets, letting $\left\{P_{+}\right\}=S_{+}$and $\left\{P_{-}\right\}=S_{-}$, we have index $\left(T_{\bar{\theta}}, P_{ \pm}\right)= \pm 1$. Indeed, choose $\epsilon>0$ such that $B_{\epsilon}\left(P_{+}\right) \cap B_{\epsilon}\left(P_{-}\right)=\varnothing$. By Sard's theorem, there exist two sequences of regular values, $\left\{\lambda\left(s_{k}\right)\right\}_{s_{k}>0}$ and $\left\{\lambda\left(t_{k}\right)\right\}_{t_{k}<0}$ such that

$$
\lambda\left(s_{k}\right) \rightarrow 1, \lambda\left(t_{k}\right) \rightarrow 1, k \rightarrow \infty, P\left(s_{k}\right) \in B_{\epsilon}\left(P_{+}\right), P\left(t_{k}\right) \in B_{\epsilon}\left(P_{-}\right) .
$$

Hence, for sufficiently large $k$,

$$
\begin{aligned}
\operatorname{deg}\left(\mathrm{id}-T_{\bar{\theta}}, B_{\epsilon}\left(P_{+}\right), 0\right) & =\operatorname{deg}\left(\mathrm{id}-T_{\bar{\theta}}, B_{\epsilon}\left(P_{+}\right), r_{k}\right) \\
& =\sum_{p \in\left(\mathrm{id}-T_{\bar{\theta}}\right)^{-1}\left(r_{k}\right)} \operatorname{sgn} \operatorname{det}\left(T_{\bar{\theta}}^{\prime}(p)\right),
\end{aligned}
$$

where $r_{k}=-\left(1-\lambda\left(s_{k}\right)\right)\left(\begin{array}{l}F \\ 0\end{array}\right)$. Notice

$$
P\left(s_{k}\right) \rightarrow P_{+}, \lambda\left(s_{k}\right)<1, \lambda\left(s_{k}\right) \rightarrow 1, s_{k}>0 .
$$


Therefore, from (19) it follows that

$$
\operatorname{index}\left(T_{\bar{\theta}}, P_{+}\right)=1 \text {. }
$$

Similarly,

$$
\operatorname{index}\left(T_{\bar{\theta}}, P_{-}\right)=-1 \text {. }
$$

Now we prove that $T$ has at least two fixed points in $A$. In the same way as in choosing $(\bar{r}, \bar{\theta})$, choose a sequence $\left\{\left(\bar{r}_{k}, \bar{\theta}_{k}\right)\right\}$ such that $\bar{\theta}_{k} \in\left(\sigma_{1}, \sigma_{2}\right), \bar{\theta}_{k} \rightarrow$ $0, k \rightarrow \infty$, and without loss of generality, let $\bar{r}_{k} \rightarrow \bar{r}_{0} \neq 0$. Set

$$
H_{k}\left(p_{k}, p, \lambda\right)=F_{\bar{\theta}_{k}}(p)-(1-\lambda) F_{\bar{\theta}_{k}}\left(p_{k}\right),
$$

where $p_{k} \in F_{\bar{\theta}_{k}}^{-1}\left(\bar{r}_{k}, 0\right)$. Then the Cauchy problem

$$
\begin{aligned}
& \frac{d x_{i}}{d s}=(-1)^{i+1} \operatorname{det}\left(H_{k}\right)_{i}^{\prime}, \quad i=1,2,3, \\
& \left(p_{k}(0), \lambda_{k}(0)\right)=\left(p_{k}, 0\right)
\end{aligned}
$$

determines a $C^{1}$ path $\left(P_{k}(s), \lambda_{k}(s)\right)$ in $\left(H_{k}\right)_{p_{k}}^{-1}(0)$. Applying the Arzela-Ascoli theorem and passing to a subsequence if necessary, we may assume $P_{k} \rightarrow P^{*}$, and for each compact interval $I$ with $\lambda_{k}(s) \leq 1$,

$$
P_{k}(s) \rightarrow P(s), \quad \lambda_{k}(s) \rightarrow \lambda(s) \text { uniformly on } I .
$$

Set

$$
S_{k_{ \pm}}=\left\{q \in \Pi A: P_{k}(s) \text { leads to } q, \text { as } \lambda_{k}(s) \rightarrow 1, s \in R_{ \pm}\right\}
$$

and

$$
D_{ \pm}=\bigcap_{k=1}^{\infty} \bigcup_{i=k}^{\infty} S_{k_{ \pm}} \text {. }
$$

Obviously, every point $p \in D_{+} \cup D_{-}$is a fixed point of $T$ in $A$. We claim that $D_{+} \cup D_{-}$contains at least two points.

Indeed, if not, then $D_{+} \cup D_{-}=\{p\}$. Since $\bar{r}_{k} \rightarrow \bar{r}_{0} \neq 0$, we have

$$
\varliminf_{k \rightarrow \infty} \sup \left\{\left|r_{k}(s)-r_{k}\left(s_{1}\right)\right|+\left|\theta_{k}(s)-\theta_{k}\left(s_{1}\right)(\bmod 2 \pi)\right|: \lambda_{k}(s), \lambda_{k}\left(s_{1}\right)<1\right\}>0 \text {. }
$$

Notice if $D_{+} \cup D_{-}=\{p\}$, then

$$
\begin{aligned}
& \theta_{k}^{\prime}(s) \neq 0, \text { for } \lambda_{k}(s)<1, \\
& \varliminf_{k \rightarrow \infty} \operatorname{meas}\left\{\theta_{k}(s): \lambda_{k}(s) \leq 1\right\} \geq 2 \pi .
\end{aligned}
$$

First, we have

$$
\varliminf_{k \rightarrow \infty} \operatorname{meas}\left\{\theta_{k}(s): \lambda_{k}(s) \leq 1\right\} \neq 0
$$

If not, without loss of generality, we assume

$$
\varliminf_{k \rightarrow \infty} \operatorname{meas}\left\{\theta_{k}(s): \lambda_{k}(s) \leq 1\right\}=0
$$

Hence $\underline{\lim }_{k \rightarrow \infty}$ meas $\left\{r_{k}(s): \lambda_{k}(s) \leq 1\right\}>0$. Since when $\lambda_{k}(s)<1, \theta_{k}(s)$ is monotonic, and if $\theta_{k}^{\prime}(s)=0$, then $\theta_{k}(s) \equiv$ a constant, and $r_{k}^{\prime}(s) \neq 0$. We have

$$
D_{+} \cup D_{-}=\bigcap_{k=1}^{\infty} \bigcup_{i=k}^{\infty}\left\{r_{k}(s): \lambda_{k}(s) \leq 1\right\} \text {. }
$$




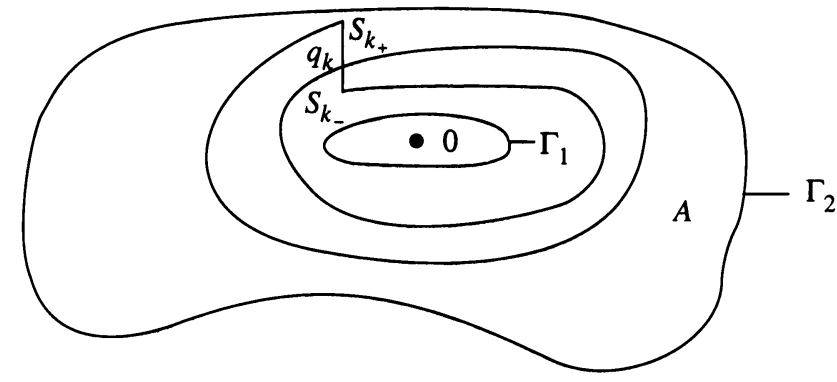

FIGURE 1

This shows

a contradiction.

$$
\operatorname{meas}\left(D_{+} \cup D_{-}\right)=\lim _{k \rightarrow \infty} \operatorname{meas}\left\{r_{k}(s): \lambda_{k}(s)<1\right\}>0,
$$

Thus, $\theta_{k}(s)$ is strictly monotonic. Since meas $\left\{\theta_{k}(s): \lambda_{k}<1\right\}>0$, by $(\mathrm{P})$ and $D_{+} \cup D_{-}=\{p\}$ we get $(22)$, and $\theta_{k}^{\prime}(s) \neq 0$. It follows that for each $k$, there exist $\theta_{k_{+}}, \theta_{k_{-}} \in R$ such that

$$
\theta_{k}(s) \rightarrow \theta_{k_{ \pm}} \text {, when } \lambda_{k}(s) \rightarrow 1, s \in R_{ \pm} \text {. }
$$

Thus,

$$
\begin{aligned}
& \left|\theta_{k_{+}}-\theta_{k_{-}}(\bmod 2 \pi)\right| \rightarrow 0,\left|\theta_{k}(s)-\theta_{0}(\bmod 2 \pi)\right| \rightarrow 0, \\
& \left|r_{k}^{*}(s)-r_{0}\right|+\left|r_{k}(s)-r_{0}\right| \rightarrow 0, \text { as } \lambda_{k}(s) \rightarrow 1, s \in R_{ \pm}, k \rightarrow \infty,
\end{aligned}
$$

where $\left(r_{0}, \theta_{0}\right)=p$. By $(22)$ and Jordan's separation theorem, for each $k$, there exists a point $q_{k}=\left(r_{k}, \theta_{k}\right)$ lying in the line segment $l_{k}$ determined by $S_{k_{+}}$and $S_{k_{-}}$, such that

$$
\operatorname{dist}\left(q_{k}, S_{k_{+}}\right)=\inf \left\{\operatorname{dist}\left(S_{k_{+}}, q\right): q \in\left(\left\{p_{k}(s)\right\} \cap l_{k}\right) \cup S_{k_{-}}\right\}
$$

See Figure 1.

Then there exists $t_{k} \in R \cup\{-\infty\}$ such that $q_{k} \in\left\{p_{k}\left(t_{k}\right)\right\}$. Let $N_{k}$ denote the line segment determined by $\left\{q_{k}\right\}$ and $S_{k_{+}}$. Thus, the curve

$$
\Gamma_{k}=\left\{P_{k}(s): \lambda_{k}(s) \leq 1, s \in\left[t_{k}, \infty\right)\right\} \cup N_{k}
$$

is a simple closed curve.

For any $\epsilon \in(0, \pi / 8)$, let

$$
\tilde{P}_{k}(s)=\left(\tilde{r}_{k}(s), \tilde{\theta}_{k}(s)\right)=P_{k}(s), \tilde{\lambda}_{k}(s)=\lambda_{k}(s) \text {, for } \theta_{k}(s) \notin\left(\theta_{0}-\epsilon, \theta_{0}+\epsilon\right) \text {. }
$$

By the Arzela-Ascoli theorem, without loss of generality, we may assume $\tilde{p}_{k}(s) \rightarrow p(s), \tilde{\lambda}_{k}(s) \rightarrow \lambda(s)$, uniformly with respect to $\theta_{k}(s) \notin\left(\theta_{0}-\epsilon, \theta_{0}+\epsilon\right)$. This shows that $(P(s), \lambda(s))$ is a loop and satisfies

$$
\lambda(s) \leq 1, T_{1}(P(s))-P(s)=(1-\lambda(s))\left(\begin{array}{c}
\bar{r}_{0} \\
0
\end{array}\right) .
$$

The set $\Omega$ is a bounded region bounded by $\{P(s)\}$. Then $\Omega$ is star shaped about the origin. Notice if $D_{+} \cup D_{-}=\{p\}$, then $\lambda(s) \not \equiv 1$. Thus

$$
\begin{aligned}
& \operatorname{meas}\left(T_{1}(\Omega)\right)>\operatorname{meas}(\Omega), \text { for } \bar{r}_{0}>0, \\
& \operatorname{meas}\left(T_{1}(\Omega)\right)<\operatorname{meas}(\Omega), \text { for } \bar{r}_{0}<0,
\end{aligned}
$$

which leads to a contradiction. This completes the proof of the theorem . 


\section{A NUMERICAL EXAMPLe to THE DUfFING EQUATIONS}

In the above section, we have established the global convergence of our homotopy. As an application, in this section, we shall give a general framework of finding periodic solutions for the Duffing equations and also shall provide a numerical example.

Consider the Duffing equations

$$
x^{\prime \prime}+F(t, x)=0
$$

where $F: R \times R \rightarrow R$ is a continuous function which is twice continuously differentiable in the second variable and $2 \pi$-periodic in the first variable. Then (25) has the equivalent form

$$
x^{\prime}=y, \quad y^{\prime}=-F(t, x) .
$$

Let $\left(x\left(t, x_{0}, y_{0}\right), y\left(t, x_{0}, y_{0}\right)\right)$ denote the unique solution of (26) with the initial value $(x(0), y(0))=\left(x_{0}, y_{0}\right)$. Then the Poincare map $T: R^{2} \rightarrow R^{2}$ defined by

$$
T\left(x_{0}, y_{0}\right)=\left(x\left(2 \pi, x_{0}, y_{0}\right), y\left(2 \pi, x_{0}, y_{0}\right)\right)
$$

is an area-preserving homeomorphism (see [29]).

Set $x=r \cos \theta, y=r \sin \theta$. Then (26) is transformed into

$$
\begin{aligned}
& r^{\prime}=r \sin \theta \cos \theta-\sin \theta F(t, r \cos \theta), \\
& \theta^{\prime}=-\sin ^{2} \theta-\frac{1}{r} \cos \theta F(t, r \cos \theta) .
\end{aligned}
$$

Denote by $\left(r\left(t, r_{0}, \theta_{0}\right), \theta\left(t, r_{0}, \theta_{0}\right)\right)$ the unique solution of (27) with the initial value $(r(0), \theta(0))=\left(r_{0}, \theta_{0}\right)$. Using the notation of the Theorem,

$$
\begin{aligned}
& r_{0}^{*}=r\left(2 \pi, r_{0}, \theta_{0}\right)=f\left(r_{0}, \theta_{0}\right), \\
& \theta_{0}^{*}=\theta\left(2 \pi, r_{0}, \theta_{0}\right)=g\left(r_{0}, \theta_{0}\right)+\theta_{0}-2 k \pi, \quad k=1,2, \ldots .
\end{aligned}
$$

To compute the periodic solutions of $(25)$, we first fix a variable $\theta_{0} \in[0,2 \pi)$, and find a point $r_{0}$ near the set $\left\{r: g\left(r, \theta_{0}\right)=0, r>0\right\}$ by Newton's method. By the parametrized Sard theorem, the choice of such a point $\left(r_{0}, \theta_{0}\right)$ is of probability one. That zero is a regular value of the homotopy

$$
H(r, \theta, \lambda)=\left(\begin{array}{c}
f(r, \theta)-r \\
g(r, \theta)
\end{array}\right)-(1-\lambda)\left(\begin{array}{c}
f\left(r_{0}, \theta_{0}\right)-r_{0} \\
0
\end{array}\right) .
$$

When $\lambda=0$, the solution of $H(r, \theta, \lambda)=0$ is $\left(r_{0}, \theta_{0}, 0\right)$; and when $\lambda \rightarrow$ 1 , any limit point $\left(r^{*}, \theta^{*}\right)$ of the solutions of (29) satisfies $f\left(r^{*}, \theta^{*}\right)=r^{*}$, $g\left(r^{*}, \theta^{*}\right)=\theta^{*}+2 k \pi, k=1,2, \ldots$

It is clear (29) is equivalent to the following initial value problem to the differential equation:

$$
\frac{d H(r(s), \theta(s), \lambda(s))}{d s}=0, \quad \lambda(0)=0 .
$$

By the discussion in section 2, to find two solutions of (25), we only need to follow two different directions to $\lambda(s)=1$ of the curve determined by (30).

Following the line of Allgower and Georg [3], we have the following algorithm. 
Algorithm 1 (Euler-Newton method).

Step 0: Given an initial point $\theta_{0} \in[0,2 \pi)$, compute $r_{0}$ such that $g\left(r_{0}, \theta_{0}\right)$ $=0$ by Newton's method, and $\lambda(0)=0$, an initial steplength $h_{0}>0, \tau \in$ $(0,1)$ and three small positive numbers $\epsilon_{1}, \epsilon_{2}, \epsilon_{3}>0$. Set $k:=0$.

Step 1: Compute a predictor direction $\eta_{k}$ :

(a) Compute a unit tangent vector $\xi_{k} \in R^{3}$;

(b) Determine the direction $\eta_{k}$ of predictor step:

If the sign of

$$
\operatorname{det}\left(\begin{array}{c}
D H\left(r_{k}, \theta_{k}, \lambda_{k}\right) \\
\xi_{k}^{T}
\end{array}\right)
$$

does not change, then $\eta_{k}=\xi_{k}$;

If the sign of

$$
\operatorname{det}\left(\begin{array}{c}
D H\left(r_{k}, \theta_{k}, \lambda_{k}\right) \\
\xi_{k}^{T}
\end{array}\right)
$$

changes, then $\eta_{k}=-\xi_{k}$;

Step 2: Compute the next iterative point $\left(r_{k+1}, \theta_{k+1}\right)$ :

(a) Compute a predictor point

$$
\left(r_{k, j}, \theta_{k, j}, \lambda_{k, j}\right)=\left(r_{k}, \theta_{k}, \lambda_{k}\right)+\tau^{j} h_{k} \eta_{k} .
$$

(b) Compute a corrector point

$$
\begin{aligned}
& \left(r_{k+1, j}, \theta_{k+1, j}, \lambda_{k+1, j}\right) \\
& \quad=\left|\left(r_{k, j}, \theta_{k, j}, \lambda_{k, j}\right)\right|-D H\left(r_{k}, \theta_{k}, \lambda_{k}\right)^{+} H\left(r_{k, j}, \theta_{k, j}, \lambda_{k, j}\right), \\
& j=0,1,2, \ldots .
\end{aligned}
$$

until $\left\|H\left(r_{k+1, j}, \theta_{k+1, j}, \lambda_{k+1, j}\right)\right\| \leq \epsilon_{1}$. Set $\left(r_{k+1}, \theta_{k+1}, \lambda_{k+1}\right)=\left(r_{k+1, j}\right.$, $\left.\theta_{k+1, j}, \lambda_{k+1, j}\right)$.

Step 3: If $\lambda_{k+1} \in\left[1-\epsilon_{2}, 1\right]$, then set $\lambda=1$ and use Newton's method to compute the periodic solutions for the Duffing equations until the iterative point $\left(r_{k}, \theta_{k}\right)$ satisfying $\left\|H\left(r_{k}, \theta_{k}, 1\right)\right\| \leq \epsilon_{3}$; else choose a new steplength $h_{k+1}>0 . k:=k+1$, go to Step 1 , where

$$
D H(r, \theta, \lambda)=\frac{d H(r, \theta, \lambda)}{(d r, d \theta, d \lambda)}
$$

and

$$
D H(r, \theta, \lambda)^{+}=D H(r, \theta, \lambda)^{T}\left(D H(r, \theta, \lambda) D H(r, \theta, \lambda)^{T}\right)^{-1}
$$

is the Moore-Penrose inverse, and $h_{k+1}$ is chosen as in [3].

Remark. By $f(r, \theta)=r(2 \pi, r, \theta)-r, g(r, \theta)=\theta(2 \pi, r, \theta)-\theta+2 k \pi, k=$ $1,2, \ldots$, we can use fourth order Runge-Kutta method to compute $f(r, \theta)$, $g(r, \theta)$ and the approximation of

$$
\frac{d f(r, \theta)}{d r}, \frac{d f(r, \theta)}{d \theta}, \frac{d g(r, \theta)}{d r}, \frac{d g(r, \theta)}{d \theta} .
$$

In the following, we give an example for the superliner Duffing equation. For the general theory of such equations, refer to [28] and [29]. Set $g(r, \theta)=$ $\theta(2 \pi, r, \theta)-\theta+2 \pi, \epsilon_{1}=0.1, \epsilon_{2}=0.01, \epsilon_{3}=10^{-5}$. Numerical results are computed by double precision operations. 


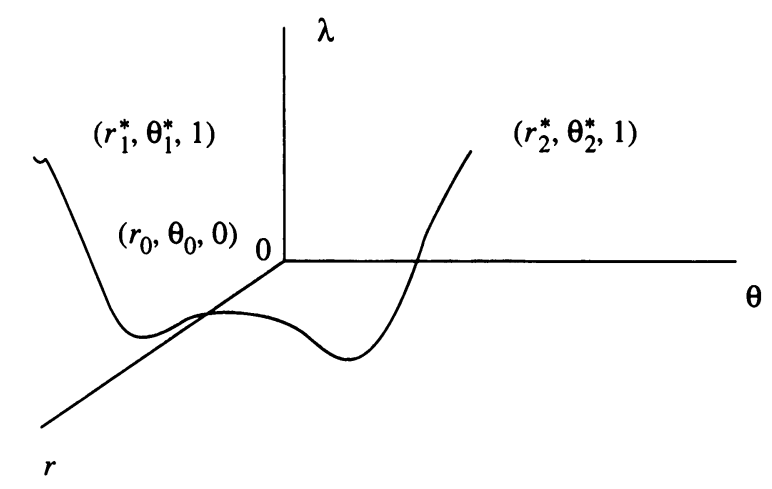

FIGURE 2. The homotopy path curve

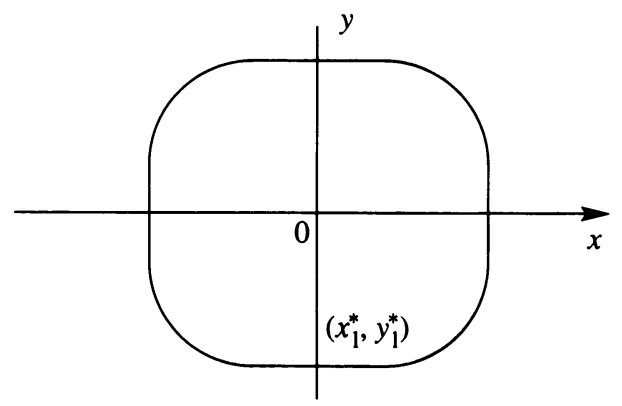

FIGURE 3

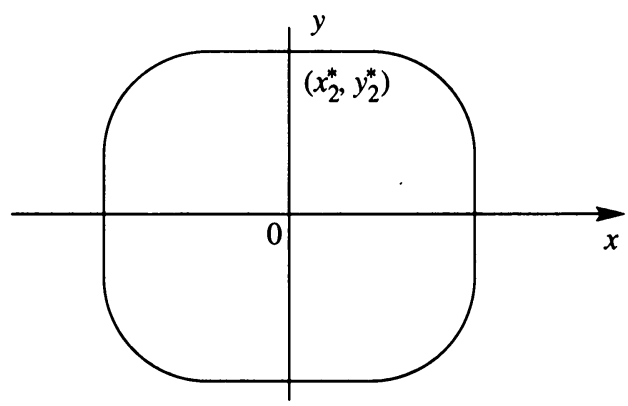

FIGURE 4

Example 1. Find at least two periodic solutions of $x^{\prime \prime}+x^{3}=(\sin t) / 10$.

By (26) and (27), Example 1 can be transformed into

$$
\begin{aligned}
& r^{\prime}=r \sin \theta \cos \theta-\sin \theta\left((r \cos \theta)^{3}-\frac{\sin t}{10}\right), \\
& \theta^{\prime}=-\sin ^{2} \theta-\frac{1}{r} \cos \theta\left((r \cos \theta)^{3}-\frac{\sin t}{10}\right) .
\end{aligned}
$$

By a simple computation, we obtain an initial value $\left(r_{0}, \theta_{0}\right)=(1.210101$, $-1.018535)$. Using homotopy (29), we can get two distinct solutions $\left(x_{1}^{*}, y_{1}^{*}\right)=$ $(0,-0.955017)$ and $\left(x_{2}^{*}, y_{2}^{*}\right)=(0,1.009132)$. In the following, Figure 2 is the homotopy path, which is generated by $(29)$ and its initial point is $\left(r_{0}, \theta_{0}\right)$; Figures 3 and 4 are two periodic solutions of Example 1, and their initial points are $\left(x_{1}^{*}, y_{1}^{*}\right),\left(x_{2}^{*}, y_{2}^{*}\right)$, respectively. Hence they are two distinct $2 \pi$-periodic solutions of the equation.

\section{ACKNOWLEDGEMENT}

The authors thank the referee for helpful comments. 


\section{REFERENCES}

1. J. C. Alexander and J. A. Yorke, The homotopy continuation method: Numerically implementable topological procedures, Trans. Amer. Math. Soc. 242 (1978), 271-284.

2. E. L. Allgower and K. Georg, Simplicial continuation methods for approximating fixed points and solutions to systems of equations, SIAM Rev. 22 (1980), 28-85.

3. York, 1990.

4. S. Alpern and V. S. Prasad, Combinatorial proofs of the Conley-Zehnder-Franks theorem on a fixed point for torus homeomorphisms, Adv. in Math. 99 (1993), 238-247.

5. __ Fixed points of area-preserving annulus homeomorphisms, Fixed Point Theory and Applications (M.A. Thera and J.B. Baillon, Eds.), Pitman Research Notes in Math., 252, 1991, pp. 1-8.

6. V. I. Arnold, Fixed points of symplectic diffeomorphisms, Proc. Sympos. Pure Math., vol. 28, Amer. Math. Soc., Providence, R.I., 1976.

7. G. D. Birkhoff, Proof of Poincare's last geometric theorem, Trans. Amer. Math. Soc. 14 (1913), 333-343.

8. __ An extension of Poincare's last geometric theorem, Acta Math. 47 (1925), 297-311.

9. __ Dynamical systems, Amer. Math. Soc. Colloq. Publ., vol. 27, Amer. Math. Soc. Providence, R.I., 1927; revised 1966; reprinted 1979.

10. $\_$Une generalisation a-n-dimensions du dernier theoreme de geometrie de Poincaré, C.R. Acad. Sci. 192 (1931), 196-198.

11. S-N. Chow, J. Mallet-Paret and J. A. Yorke, Finding zeros of maps: Homotopy methods that are constructive with probability one, Math. Comp. 143 (1978), 887-899.

12. C. Conley and E. Zehnder, The Birkhoff-Lewis fixed point theorem and a conjecture of V.I. Arnold, Invent. Math. 73 (1983), 33-49.

13. C. Conley and E. Zehnder, Morse-type index theory for flows and periodic solutions for Hamiltonian equations, Comm. Pure Appl. Math. 37 (1984), 207-253.

14. M. A. Del Pino, R. Manaserich and A. E. Murna, On the number of $2 \pi$-periodic solutions for $u^{\prime \prime}+g(u)=s(1+h(t))$ using the Poincare-Birkhoff theorem, J. Differential Equations 95 (1992), 240-258.

15. R. Dieckerhoff and E. Zehnder, An "a-priori" estimate for oscillatory- equations, Lecture Notes in Math., vol. 1125, Springer, 1985.

16. T. Ding, Nonlinear oscillations at point of resonance, Sci. Sinica Ser. A 25 (1982), 918-931.

17. _ An infinite class of periodic solutions of periodically perturbed Duffing equations at resonance, Proc. Amer. Math. Soc. 86 (1982), 47-54.

18. T. Ding and W. Ding, Resonance problem for a class of Duffing equations, Chinese Ann. Math. Ser. B 6 (1985), 427-432.

19. T. Ding and F. Zanolin, Time-maps for the solvability of periodically perturbed nonlinear Duffing equations, Nonlinear Analysis, TMA, 1991.

20. W. Ding, Fixed points of twist mappings and periodic solutions of ordinary differential equations, Acta Math. Sinica (Chinese) 25 (1982), 227-235.

21. A A generalization of the Poincaré-Birkhoff theorem, Proc. Amer. Math. Soc. 88 (1983), 341-346.

22. M. Flucher, Fixed points of measure preserving torus homeomorphisms, Manuscripta Math. 68 (1990), 271-293.

23. A. Fonda, R. Manaserich and F. Zanolin, Subharmonic solutions for some second-order differential equations with singularities, SIAM J. Math. Anal. 24 (1993), 1294-1311.

24. J. Franks, Recurrence and fixed points of surface homeomorphisms, Ergodic Theory Dynamical Systems 8 (1988), 99-107.

25. J. Franks, Generalizations of the Poincaré-Birkhoff theorem, Ann. Math. 128 (1988), 139151. 
26. C. B. Garcia and W. I. Zangwill, An approach to homotopy and degree theory, Math. Oper. Res. 4 (1979), 390-405.

27. __ Pathways to solution, fixed points, and equilibria, Prentice-Hall, Englewood Cliffs, NJ, 1981.

28. P. Hartman, On boundary value problems for superlinear second order differential equations, J. Differential Equations 26 (1977), 37-53.

29. H. Jacobowitz, Periodic solutions of $x^{\prime \prime}+f(x, t)=0$ via the Poincaré-Birkhoff theorem (and Corrigendum: The existence of the second fixed point), J. Differential Equations 20 (1976), 37-52; 25 (1977), 148-149.

30. H. B. Keller, Global homotopies and Newton methods, Recent Advances in Numerical Analysis (C. de Boor and G. Golub, Eds.), Academic Press, New York., 1978, pp. 73-94.

31. R. B. Kellogg, T. Y. Li and J. A. Yorke, A constructive proof of the Brouwer fixed point theorem and computational results, SIAM J. Numer. Anal. 13 (1976), 473-483.

32. M. Levi, KAM theory for particles in periodic potentials, Ergodic Theory Dynamical Systems 10 (1990), 777-785.

33. Li Yong and Lu Xianrui, Continuation theorems to boundary value problems, J. Math. Anal. Appl. 188 (1994).

34. B. Liu, Boundedness for solutions of nonlinear Hill's equations with periodic forcing terms via Moser's twist theorem, J. Differential Equations 79 (1989), 304-315.

35. _ An existence theorem for harmonic solutions of semi-linear Duffing equations, Acta Math. Sinica 34 (1991), 165-170.

36. G. Morris, A case of boundedness in Littlewood's problem on oscillatory differential equations, Bull. Austral. Math. Soc. 14 (1976), 71-93.

37. J. Moser, On invariant curves of area-preserving mapping of annulus, Nachr. Akad. Wiss. Gottingen Math. Phys. K. 2 (1962), 1-20.

38. H. Poincaré, Sur un theoreme de geometrie, Rend. Circ. Mat. Palermo 33 (1912), 375-407.

39. S. Smale, A convergent process of price adjustment and global Newton methods, J. Math. Econom. 3 (1976), 1-14.

40. L. T. Watson and M. R. Scott, Solving spline-collocation approximations to nonlinear twopoint boundary value problems by a homotopy method, Appl. Math. Comput. 24 (1987), 333-357.

Department of Mathematics, Jilin University, Changchun 130023, P.R. of China 